LA W RENCE LIVERMORE N A TIO NAL LABORATORY
An application of Fleck effective scattering to the difference formulation for photon transport

Frank Daffin

October 18, 2006 
This document was prepared as an account of work sponsored by an agency of the United States Government. Neither the United States Government nor the University of California nor any of their employees, makes any warranty, express or implied, or assumes any legal liability or responsibility for the accuracy, completeness, or usefulness of any information, apparatus, product, or process disclosed, or represents that its use would not infringe privately owned rights. Reference herein to any specific commercial product, process, or service by trade name, trademark, manufacturer, or otherwise, does not necessarily constitute or imply its endorsement, recommendation, or favoring by the United States Government or the University of California. The views and opinions of authors expressed herein do not necessarily state or reflect those of the United States Government or the University of California, and shall not be used for advertising or product endorsement purposes.

This work was performed under the auspices of the U.S. Department of Energy by University of California, Lawrence Livermore National Laboratory under Contract W-7405-Eng-48. 


\title{
An application of Fleck effective scattering to the difference formulation for photon transport
}

\author{
Frank Daffin \\ University of California \\ Lawrence Livermore National Laboratory \\ Livermore, California 94550 \\ E-mail: daffin1 @1lnl.gov
}

\begin{abstract}
We introduce a new treatment of the difference formulation[1] for photon radiation transport without scattering in 1-d slab geometry that is closely analogous to that of Fleck and Cummings[2] for the traditional formulation. The resulting form is free of implicit source terms and has the familiar effective scattering of the field of transport.
\end{abstract}

Key Words: difference formulation; Monte Carlo methods; photon transport

\section{INTRODUCTION}

Symbolic-Implicit Monte Carlo (SIMC) treatments[3] of the difference formulation of photon transport[1] have demonstrated impressive levels of noise reduction. As it has been applied to the difference formulation in local thermodynamic equilibrium (LTE), the SIMC method enhances the numerical stability of those calculations at the expense of a non-linear system solve at each time step. For well-conditioned systems discretized into relatively few zones, the computational effort is typically dominated by particle tracking; the expense of the non-linear solver is inconsequential, and SIMC applied to either the standard or the differnece formulation enables the use of large time steps while maintaining numerical stability. This can reduce overall computation time.

Notwithstanding considerations of scattering and connectivity in higher dimensions, for problems of sufficiently large spacial extent the matrix to be inverted will be band-diagonal, the width of which is limited by int $(c \delta t / \delta x)+1$, where $c$ is the propagation speed, $\delta t$ is the time step size, and $\delta x$ is the size of the zones used. Additionally, optically thick problems guarantee that the reach of influence of Monte Carlo particles from symbolic sources will be limited by attenuation, especially when techniques are utilized for eliminating particles of insignificant weight. Either way, the band-limiting tends to ease the iterative burden by reducing the influence of the non-linear portions of the system solve. However, if one considers the costs associated with inverting the large matrices storing Monte Carlo sums for a finely-discretized system, then one may find that multi-dimensional, high-fidelity calculations using SIMC to be impractical. 
To improve the numerical stability of the Monte Carlo solution of the standard photon transport equation, Fleck and Cummings[2] introduced a method of that converts a portion of the absorption into an artificial scattering term, weakening the coupling between the radiation transport and material energy equations. While the treatment, now called Implicit Monte Carlo (IMC), is not perfect[4][5] and can be costly in optically thick problems where there are many effective scattering events, it is unconditionally stable and does not require the extra storage or computational burden of the solution of the non-linear system.

\section{FLECK-LIKE TREATMENT OF THE DIFFERENCE FORMULATION}

For optically thick systems in LTE, the departure of the specific photon intensity, $I$, from the planck field, $B$, is small[1]. The difference formulation takes advantage of this by transforming from the photon field, which is large in these systems, to the difference field, $D=I-B$. It is the smallness of the difference field that we think will work in favor of the IMC method.

In the SIMC treatments of the difference formulation currently known to the author[6], the reduction in noise is due to the small size of the field and freedom from having to sample the material's emissivity for emission frequency, a task made difficult by the rich structure in frequency the function exhibits for real materials. We believe similar benefits may be realized in an IMC treatment, since the size of the field-determined by the physics of the problem and not the solution method-should be small. With fewer Monte Carlo particles (samples) of the field required in the calculation to achieve a desired level of noise, there should be fewer scattering events to calculate too. We conjecture that two of IMC's disadvantages in optically thick problems, namely it's Monte Carlo noise and frequent effective scattering, may be mitigated by an analogous treatment of the difference formulation.

The source terms of the difference formulation are treated implicitly in [6] to improve numerical stability, and it is this implicit treatment that leads to the system of non-linear equations that must be solved for the temperatures of the cells at the end of each time step. Beginning with the difference formulation for photon transport without scattering in 1-d slab geometry,

$$
\frac{1}{c} \frac{\partial D}{\partial t}+\mu \frac{\partial D}{\partial x}=-\sigma D-\left(\frac{1}{c} \frac{\partial}{\partial t}+\mu \frac{\partial}{\partial x}\right) B
$$

we closely follow the development of the IMC treatment of the standard photon transport formulation[2].

The black body field $B$ can be expressed as $\frac{1}{2} c u_{r}$, where $u_{r}=a T^{4}$ is the radiation energy density. Closely following Fleck[2], the energy density equation is written as

$$
\frac{\partial u_{r}}{\partial t}=\beta \sigma\left(\int_{-1}^{1} I d \mu+c u_{r}\right)+\beta S
$$

where $S$ is an external energy source and

$$
\beta=\frac{\partial u_{r}}{\partial u_{m}}
$$


with $u_{m}$ being the material energy density. Integrating both sides of Eq. 2 and taking the appropriate time-centered values, leads to an expression for the radiation energy density

$$
u_{r}^{\gamma}=\frac{\alpha \beta \sigma \Delta t}{1+\alpha \beta \sigma c \Delta t} \int_{-1}^{1} I^{\lambda} d \mu+\frac{u_{r}^{n}}{1+\alpha \beta \sigma c \Delta t}+\frac{\alpha \beta \Delta t S^{\gamma}}{1+\alpha \beta \sigma c \Delta t}=\frac{2 B^{\gamma}}{c}
$$

where the superscripts $\lambda, \gamma$ and the factor $\alpha$ define the time-centering of the mean values of $I, S, B$ and $u_{r}$, e.g.:

$$
u_{r}^{\gamma}=\alpha\left(u_{r}^{n+1}-u_{r}^{n}\right)+u_{r}^{n}
$$

with $n$ being the time-integration cycle index and $\Delta t=t_{n+1}-t_{n}$. Solving for $B^{\gamma}$ gives

$$
B^{\gamma}=\frac{\frac{1}{2} c \alpha \beta \sigma \Delta t}{1+\alpha \beta \sigma c \Delta t} \int_{-1}^{1} I^{\lambda} d \mu+\frac{B^{n}}{1+\alpha \beta \sigma c \Delta t}+\left(\frac{\frac{1}{2} c \alpha \beta \Delta t}{1+\alpha \beta \sigma c \Delta t}\right) S^{\gamma}
$$

Substituting this expression for $B$ into Eq. (1) gives

$$
\begin{aligned}
& \frac{1}{c} \frac{\partial D}{\partial t}+\mu \frac{\partial D}{\partial x}=-\sigma D- \\
&\left(\frac{1}{c} \frac{\partial}{\partial t}+\mu \frac{\partial}{\partial x}\right)\left(\frac{\frac{1}{2} c \alpha \beta \sigma \Delta t}{1+\alpha \beta \sigma c \Delta t} \int_{-1}^{1} I^{\lambda} d \mu+\frac{B^{n}}{1+\alpha \beta \sigma c \Delta t}+\frac{\frac{1}{2} c \alpha \beta \Delta t S^{\gamma}}{1+\alpha \beta \sigma c \Delta t}\right) \\
&=-\sigma D-\left(\frac{1}{c} \frac{\partial}{\partial t}+\mu \frac{\partial}{\partial x}\right)\left(\frac{\frac{1}{2} c \alpha \beta \sigma \Delta t}{1+\alpha \beta \sigma c \Delta t} \int_{-1}^{1} I^{\lambda} d \mu\right)- \\
& \frac{1}{1+\alpha \beta \sigma c \Delta t}\left(\frac{1}{c} \frac{\partial}{\partial t}+\mu \frac{\partial}{\partial x}\right) B^{n}-\frac{\frac{1}{2} c \alpha \beta \Delta t}{1+\alpha \beta \sigma c \Delta t}\left(\frac{1}{c} \frac{\partial}{\partial t}+\mu \frac{\partial}{\partial x}\right) S^{\gamma}
\end{aligned}
$$

On the right-hand side, the term

$$
\left(\frac{1}{c} \frac{\partial}{\partial t}+\mu \frac{\partial}{\partial x}\right)\left(\frac{\frac{1}{2} c \alpha \beta \sigma \Delta t}{1+\alpha \beta \sigma c \Delta t} \int_{-1}^{1} I^{\lambda} d \mu\right)
$$

may be written as

$$
\left(\frac{\frac{1}{2} c \alpha \beta \sigma \Delta t}{1+\alpha \beta \sigma c \Delta t}\right)\left[\left(\frac{1}{c} \frac{\partial}{\partial t}+\mu \frac{\partial}{\partial x}\right) \int_{-1}^{1} I^{\lambda} d \mu\right]
$$

Moving the streaming operator within the integral

$$
\left(\frac{\frac{1}{2} c \alpha \beta \sigma \Delta t}{1+\alpha \beta \sigma c \Delta t}\right)\left[\int_{-1}^{1}\left(\frac{1}{c} \frac{\partial}{\partial t}+\mu \frac{\partial}{\partial x}\right) I^{\lambda} d \mu\right]
$$

or

$$
\left(\frac{\frac{1}{2} c \alpha \beta \sigma \Delta t}{1+\alpha \beta \sigma c \Delta t}\right)\left[\int_{-1}^{1} \frac{1}{c} \frac{\partial I^{\lambda}}{\partial t}+\frac{1}{\mu} \frac{\partial I^{\lambda}}{\partial x} d \mu\right]
$$

The integrand looks familiar; it's just part of the standard transport equation:

$$
\frac{1}{c} \frac{\partial I}{\partial t}+\mu \frac{\partial I}{\partial x}=-\sigma(I-B)=-\sigma D
$$


where $D$ is the difference field in the difference formulation. Thus, the term now becomes

$$
\left(\frac{\frac{1}{2} c \alpha \beta \sigma \Delta t}{1+\alpha \beta \sigma c \Delta t}\right)\left[\int_{-1}^{1}-\sigma D^{\lambda} d \mu\right]
$$

which looks like an effective scattering term for the $D$-field with scattering cross-section

$$
\sigma_{s}=\left(\frac{c \alpha \beta \sigma \Delta t}{1+\alpha \beta \sigma c \Delta t}\right) \sigma
$$

The reader will recognize this as the same effective scattering cross-section that appears in the transport equation in the work by Fleck and Cummings[2]. The term

$$
\frac{1}{1+\alpha \beta \sigma c \Delta t}\left(\frac{1}{c} \frac{\partial}{\partial t}+\mu \frac{\partial}{\partial x}\right) B^{n}
$$

is close to the original form for the new source terms in the difference formulation in LTE [6], but here there are no implicit terms $B^{n+1}$. The last term is the contribution from external sources,

$$
\frac{\frac{1}{2} c \alpha \beta \Delta t}{1+\alpha \beta \sigma c \Delta t}\left(\frac{1}{c} \frac{\partial}{\partial t}+\mu \frac{\partial}{\partial x}\right) S^{\gamma}
$$

which is usually known or specified. The transport equation now has the form

$$
\begin{aligned}
\frac{1}{c} \frac{\partial D}{\partial t}+\mu \frac{\partial D}{\partial x}=-\sigma D+\frac{1}{2} \sigma_{s} \int_{-1}^{1} D^{\lambda} & d \mu-\frac{1}{1+\alpha \beta \sigma c \Delta t}\left(\frac{1}{c} \frac{\partial}{\partial t}+\mu \frac{\partial}{\partial x}\right) B^{n} \\
& -\frac{1}{2}\left(\frac{c \alpha \beta \Delta t}{1+\alpha \beta \sigma c \Delta t}\right)\left(\frac{1}{c} \frac{\partial}{\partial t}+\mu \frac{\partial}{\partial x}\right) S^{\gamma}
\end{aligned}
$$

which is free of implicit terms, but has an effective scattering term for the $D$-field.

As $\Delta t \rightarrow \infty, \sigma_{s} \rightarrow \sigma$ and the transport equation becomes

$$
\frac{1}{c} \frac{\partial D}{\partial t}+\mu \frac{\partial D}{\partial x}=-\sigma D+\frac{1}{2} \sigma \int_{-1}^{1} D^{\lambda} d \mu-\frac{1}{2}\left(\frac{1}{\sigma}\right)\left(\frac{1}{c} \frac{\partial}{\partial t}+\mu \frac{\partial}{\partial x}\right) S^{\gamma}
$$

Note the absence of source terms from the streaming operator on $B$ :

$$
\left(\frac{1}{c} \frac{\partial}{\partial t}+\mu \frac{\partial}{\partial x}\right) B
$$

the new source terms of the difference formulation. The $1 / \Delta t$-dependence of this source term in Eq.17 may lead to an unphysical decoupling between the material energy and the difference field for large time-step sizes, a behavior observed in the IMC method applied to the standard formulation[4]. As $\Delta t \rightarrow 0, \sigma_{s} \rightarrow 0$ and we recover the original difference formulation of the radiation transport equation.

$$
\frac{1}{c} \frac{\partial D}{\partial t}+\mu \frac{\partial D}{\partial x}=-\sigma D-\left(\frac{1}{c} \frac{\partial}{\partial t}+\mu \frac{\partial}{\partial x}\right) B
$$

\section{CONCLUDING REMARKS}

We have shown how the simple application of methodology used in [2] to improve the numerical stability of non-linear radiation transport has eliminated symbolic source terms 
in the difference formulation. But the old aphorism "there is no free lunch" holds here too: one must still deal with the scattering of the $D$-field and all the well-known problems it invites (radon-walk techniques [7] may reduce the burden caused by scattering) as well as the possible problems with the new $1 / \Delta t$-dependence of the source terms for large time steps. It would be interesting to see if an analysis similar to [4] could be applied. Nonetheless, it is our hope that the relatively few Monte Carlo samples required of the difference formulation will mean fewer scattering events for the $D$-field in some of the most challenging problems for the standard formulation-namely optically thick systems in LTE. Further, since the method is free of a system solve, there may be a net savings in computational effort over an SIMC implementation of the difference formulation when one tries to solve problems composed of many cells.

We close by noting that Nick Gentile of this laboratory and Richard Smedley-Stevenson of AWE are busy mining a similar vein.

\section{REFERENCES}

1. Abraham Szöke and Eugene D. Brooks III, The Transport Equation in Optically Thick Media, Journal of Quantitative Spectroscopy \& Radiative Transfer, Vol. 91, 2005, pp. 95-110.

2. J. A. Fleck, Jr and J. D. Cummings, An implicit Monte Carlo Scheme for Calculating Time and Frequency Dependent Non Linear Radiation Transport, Journal of Computational Physics, Vol. 8, Issue 3, December 1971, pp. 313-342.

3. Eugene D. Brooks III, Symbolic Implicit Monte Carlo, Journal of Computational Physics, Vol. 83, No. 2, August 1989, pp. 433-446.

4. Edward W. Larsen and Bertrand Mercier, Analysis of a Monte Carlo Method for Nonlinear Radiative Transfer, Journal of Computational Physics, Vol. 71, Issue 1, July 1987, pp. 50-64.

5. Jeffery D. Densmore and Edward W. Larsen, Asymptotic Equilibrium Diffusion Analysis of Time-Dependent Monte Carlo Methods for Grey Radiative Transfer, Journal of Computational Physics, Vol. 199, Issue 1, September 2004, pp. 175-204.

6. Eugene D. Brooks III, Michael Scott McKinley, Frank Daffin and Abraham Szöke, Symbolic Implicit Monte Carlo Radiation Transport in the Difference Formulation: a Piecewise Constant Discretization, Journal of Computational Physics, Vol. 205, Issue 2, May 2005, pp. 737-754.

7. J. A. Fleck, Jr. and E. H. Canfield, A Random Walk Procedure for Improving the Computational Efficiency of the Implicit Monte Carlo Method for Nonlinear Radiation Transport, Journal of Computational Physics, Vol. 54, Issue 3, June 1984, pp. 508-523. 